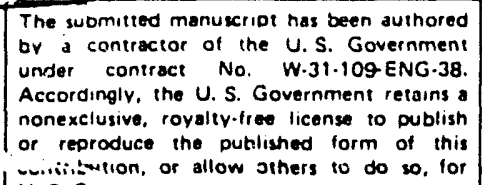

\title{
BUBBLE CHAMBER MEASUREMENTS OF CASCADE TIMES IN MESIC ATOMS
}

T. Fields

Argonne National Laboratory, Argonne, IL 60439

This paper describes the pioneering role of the bubble chamber in measuring interesting atomic physics quantities such as the cascade time in mesic hydrogen and helium atoms.

\section{INTRODUCTION}

Experiments to study the nuclear absorption of slow negatively charged particles (pions, muons, kauns, antiprotons, etc) were first performed about 50 years ago. These negative particles are normally captured into a bound atomic orbit before the nuclear absorption occurs. Consequently, atomic physics processes play a central role in determining the key properties of the initial state of the nuclear absorption reaction (angular momentum, parity, Coulomb binding energy, and wave function at the nucleus).

This note describes how early bubble chamber experiments were able to provide striking new information about atomic processes affecting negative particles stopping in liquid hydrogen and liquid helium. The first such bubble chamber measurements were reported in the early 1960's, and used pion beams from synchrocyclotrons. Some important impacts of these and other bubble chamber measurements of atomic processes upon later developments will also be described briefly.

\section{CASCADE TIME OF PIMINUS IN LIQUID HYDROGEN}

The range of a muon from the decay of a pion at rest is about one centimeter in bubble chamber hydrogen. Such a decay can be easily identified by the unique length of the muon track. The principle of a cascade time measurement is to determine the number of $\pi \rightarrow \mu$ decays which are consistent with pion decay at rest, using negative pions which come to rest (and are then absorbed) in a bubble chamber. The number of such decays will be proportional to the mean time which the pion spends at low velocity (as part of a $\pi$-p atom) before being absorbed by the proton, provided that the cascade time is much shorter than the free pion decay lifetime.

Fig 1, taken from ref 1 , shows the bubble chamber data as well as the kinematics of $\pi \rightarrow \mu$ decay in flight. In the data of Fig 1, two events having pion decays with $\beta(\pi)<0.01$ can be seen. If we define the cascade time $\tau$ as the mean time for the pion to go from $\beta=0.01$ to nuclear capture, then these data yield $\tau=1.2_{-0.9}^{+1.2} \times 10^{-12}$ sec. A later experiment [2] with higher statistics obtained $\tau=2.3 \pm 0.6 \times 10^{-12}$ sec.

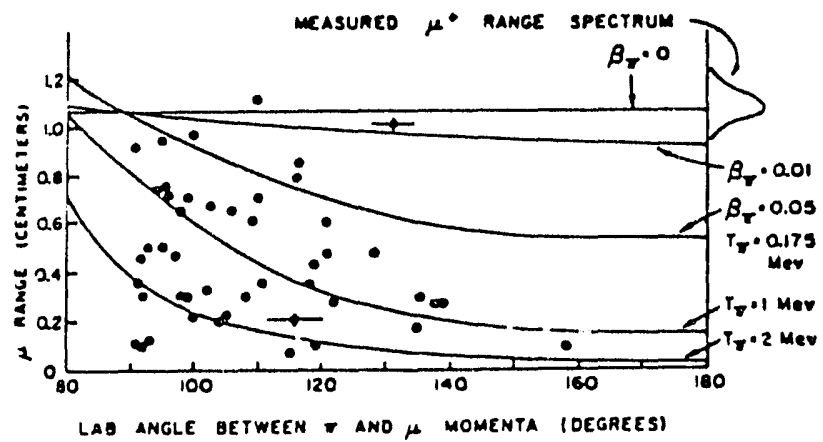

FIG. 1. Kinematics of $\pi-\mu$ decay in night. Each dot represents one $\pi \rightarrow \mu$ decay found in pictures con:aining $80000 \pi^{-}$stops in liquid hydrogen.

This result is aboui 2 orders of magnitude smaller than had been predicted by Wightman [3] in 1949. His calculation assumed that nuclear capture would occur mainly from the

To appear in the Proceedings of the Conference on the Bubble Chamber and its Contributions to Particle Physics, CERN, Geneva, Switzerland, 1993. 
1S ground state of the $\pi p$ atom, after a radiative cascade through a sequence of circular atomic orbits.

It was pointed out by Day, Snow, and Sucher [4], following a suggestion by Madansky [4], that, since the $\pi \mathrm{p}$ atom is small and electrically neutral,in its collisions with other atoms it would move through regions of high atomic electric field and would undergo Stark effect mixing of its atomic levels. The Stark mixing of l>0 states with $l=0$ states would lead to nuclear absorption from $S$ states of principal quantum number $n \geq 4$ within a short time $[5,6]$ of about $4 \times 10^{-12} \mathrm{sec}$, which is close to the measured cascade time.

Thus the bubble chamber results became the experimental basis for a new model of the pion cascade process in hydrogen which implied the dominance of $S$ state capture and the absence of $\mathrm{K}$ and $\mathrm{L} \mathrm{x}$-rays.

\section{CASCADE TIME OF PIONS IN LIQUID HELTUM}

Using the method described above, two helium bubble chamber experiments $[7,8]$ measured the piminus cascade time in liquid helium to be $3.2 \pm 0.6 \times 10^{-10} \mathrm{sec}$. This is two orders of magnitude longer than for hydrogen, and seemed to imply that the Stark effect collisional mixing phenomenon is much reduced or absent in helium. Of course, a lesser Stark effect might be expected, since the $\pi$ He system has a net positive charge. However, in fact, atomic physics calculations [9] showed that Stark mixing, though less than for hydrogen, would be expected to reduce the cascade time to a value much less than this experimental result.

A possible resolution of this puzzle was promptly suggested by Condo [10]. He pointed out the possibility that a few percent of the pions could be trapped in metastable circular orbits of large principal quantum nurnber, which would increase the mean cascade time substantially.

\section{CASCADE TIME OF KAONS IN LIQUID HELIUM}

A similar, even larger discrepancy between prediction and experiment for helium was later found when measurements $[11,12]$ were made of the kaon mean cascade time in helium. This discrepancy, augrnented by new information on kaon mesic xray intensities [13], inspired additional calculations $[13,14]$ whose results seemed to support the trapping hypothesis of Condo [10]. Russell [14] also suggested that such a hypothesis could be tested by stopping antiprotons in liquid helium and searching for delayed annihilations.

\section{SUBSEQUENT DEVELOPMENTS}

There is too little space in this paper to fully describe the ways in which the bubble chamber measurements of cascade time and other parameters of atomic processes have influenced later developments. I shall only list four examples:

- Strong interaction physics - Quantum numbers of initial states for meson and antiproton absorption at rest by hydrogen and helium are important for spin-parity determinations and for dynamical analyses. Similarly, as mentioned above, consistency is required between cascade time measurements and experiments which measure the yields of atomic $x$-rays from mesic and protium atoms.

- Muon catalyzed fusion - The original experimental discovery and understanding of this process by Alvarez et al. [15] has been described at this conference by M. L. Stevenson. That understanding depended directly upon atomic physics issues such as muon transfer from $\mu \mathrm{p}$ to $\mu \mathrm{d}$ atoms as well as the formation of the $\mu$ pd molecular ion. Important advances in muon catalyzed fusion have been made in recent years [16], building upon the suggestion of Vesman [17] that the observed large formation rate of the $\mu \mathrm{dd}$ molecular ion could be due to the existence of a resonant molecular state. 
This unexpectedly large formation rate was first seen by two early bubble chamber experiments $[18,19]$. Later cloud chamber data [20] indicated the resonance possibility.

- Muon absorption by hydrogen - The fundamental weak interaction process of $\mu+p \rightarrow v+n$ can be measured for negative muons stopped in hydrogen. However, a quantitative experiment requires knowledge of the atomic/molecular state of the muon, as well as being sure that the muon was not transferred to an impurity nucleus (as did happen in the process in which muon catalyzed fusion was originally discovered [15]).

- Antiproton trapping in liquid helium - A recent counter experiment [21] at KEK has observed that about $3.6 \%$ of antiprotons stopping in liquid helium are indeed trapped in long-lived metastable states, with lifetimes before annihilation of up to 3 microseconds. This is a striking confirmation of Russell's suggestion[14] and of Condo's interpretation [10] of the bubble chamber measurements of cascade times.

\section{SUMMARY}

The examples reviewed in this paper show that the bubble chamber technique was successfully used for measuring a number of important quantities relating to the atomic physics of mesic atoms and molecules. The early bubble chamber experiments yielded a rich harvest of atomic physics puzzles and surprises.

This work was supported by the U.S. Department of Energy, High Energy Physics Division, under Contract No. W-31-109-ENG38.

\section{REFERENCES}

1. T.H. Fields, G.B. Yodh, M. Derrick and J.G. Fetkovich, Phys. Rev. Lett. 5 (1960) 69.
2. J. Doede, R. Hildebrand, M. Israel, and M. Pyka, Phys. Rev. 129 (1963) 2808.

3. A.S. Wightman, Princeton University Thesis, 1949 (unpublished); Phys. Rev. 77 (1950) 521.

4. T.B. Day, G.A. Snow, and J. Sucher, Phys. Rev. 3 (1959) 61; and Phys. Rev. 118 (1960) 864.

5. J.E. Russell and G.L. Shaw, Phys. Rev. Lett. 4 (1960) 369.

6. M. Leon and H.A. Bethe, Phys, Rev. 127 (1952) 676.

7. J.G. Ketkovich and E.G. Pewitt, Phys. Rev. Lett. 11 (1963) 290.

8. M.M. Block, T. Kikuchi, D. Koetke, J. Kopelman, C.R. Sun, R. Walker, G. Culligan, V.L. Telegdi, and R. Winston, Phys. Rev. Lett. 11 (1963) 301.

9. T.B. Day, Nuovo Cimento 18 (1960) 381.

10. G.T. Condo, Phys. Lett. 9 (1964) 65.

11. M.M. Block, J.B. Kopelman, and C.R. Sun, Phys. Rev. 140 (1965) B143.

12. J.G. Fetkovich, J. McKenzie, B.R. Riley, I.-T. Wang, K. Bunnell, M. Derrick, T. Fields, L.G. Hyman, and G. Keyes, Phys. Rev. D2 (1970) 1803.

13. S. Berezin, G. Burleson, D. Eartly, A. Roberts, and T. White, Phys. Lett. 30B (1969) 27.

14. J.E. Russell, Phys. Rev. Lett. 23 (1969) 63.

15. L.W. Alvarez, H. Bradner, F.S. Crawford, Jr., P. Falk-Vairant, M.L. Good, J.D. Gow, A.H. Rosenfeld, F. Solmitz, M.L. Stevenson, H.K. Ticho, and R.D. Tripp, Phys. Rev. 105 (1957) 1127. 
16. For a review, see W. Breunlich, P. Kammel, J. Cohen and M. Leon, Ann.

Rev. Nucl. Part. Sci. (1989) 311.

17. E. Vesman, JETP Lett. 5 (1967) 91.

18. J. Fetkovich, T. Fields, G. Yodh, and M. Derrick, Phys. Rev. Lett. 4 (1960) 570.

19. J.H. Doede, Phys. Rev. 132 (1963) 1782.

20. V. Dzhelepov, P.F. Ermolov, V.I. Moskalev, and V.V. Kil'Chenkov, Soviet Physics JETP 23 (1966) 820.

21. M. Iwasaki, S.N. Nakamura, K. Shigatki, Y. Shimizu, H. Tamura, T. Ishikawa, and R.S. Hayano, Phys. Rev. Lett. 67 (1991) 1246.

\section{DISCLAIMER}

This report was prepared as an account of work sponsored by an agency of the United States This employees, makes any warranty, express or implied, or assumes any legal liability or responsibility for the accuracy, completeness, or usefulness of any information, apparatus, product, or bility for the accuracy, or represents that its use would not infringe privately owned rights. Referprocess disclosed, or reprif commercial product, process, or service by trade name, trademark, ence herein to any specific commercial product, process, or soces imply its endorsement, recommendation, or favoring by the United States Government or any agency thereof. The views mendation, or favoring by the United hein do not necessarily state or reflect those of the United States Government or any agency thereof. 

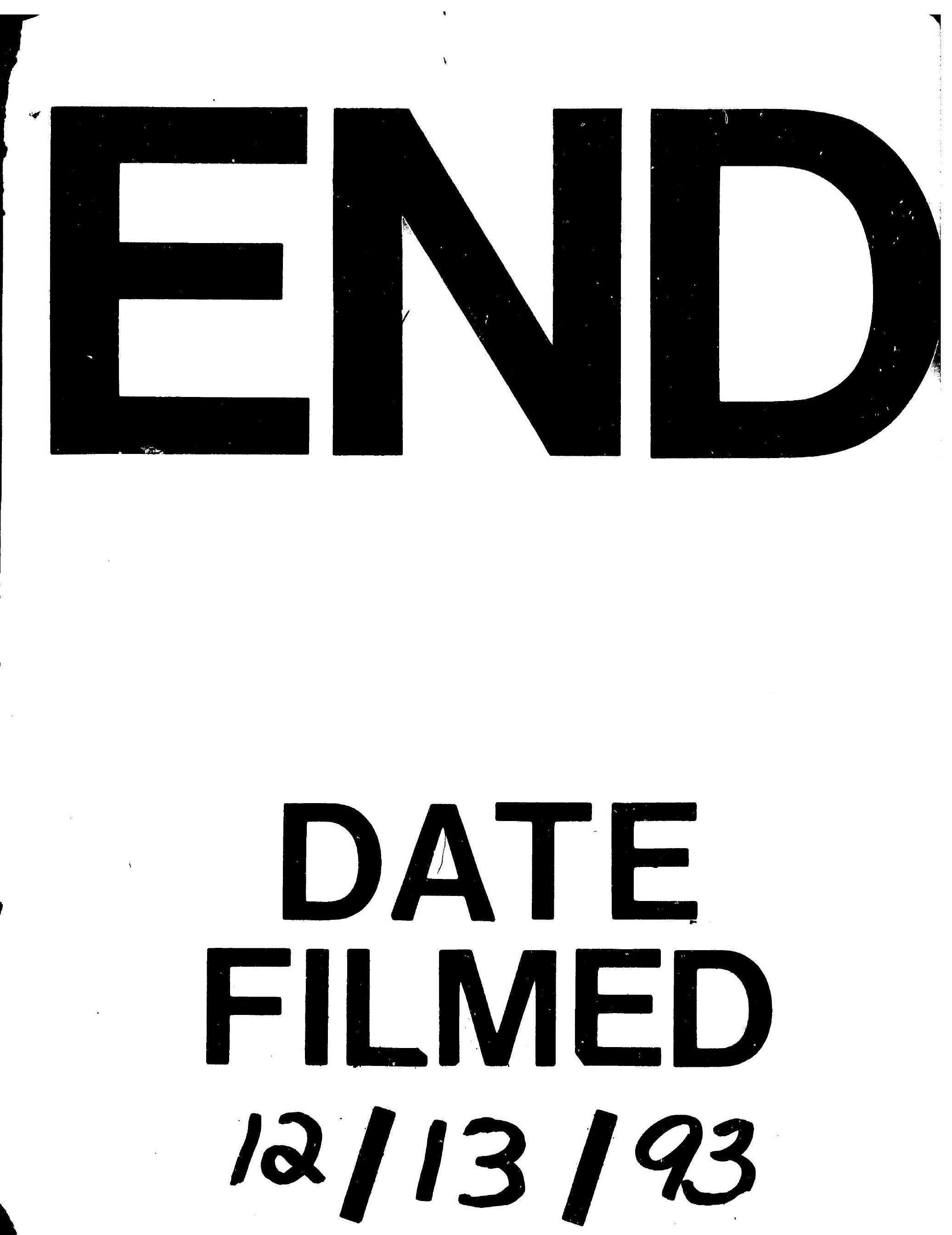
